\title{
Gottesstaat und Götterstaat unter den vorchristlichen Germanen
}

\author{
Von ÅKE V. STRÖM
}

Hjalpi mér svá Freyr ok

Njordr ok hinn almáttki óss.
Hilf mir so Frey und Njord und der allmächtige Ase.

(Lnb. H., Kap. 268)

So lautet, dem Ulfljot-Gesetz gemäß, die Eidformel am isländischen Gesetz-Ding (log ping), das Gericht und Völkerversammlung zugleich war. Die Formel muß uralt sein. Gerade nennt sie als Helfer, wie in Indien, die dioskurischen Brüder, die in alten Zeiten Nirdir, Plur. von Njordr, genannt wurden (É́rarinn máhl. Str. 5, Hávarär halti Str. $7^{1}$ ), nachmals aber Vater und Sohn (Njordr und Frey) geworden sind - und dazu den mächtigen, rechtspfiegenden Gott, ohne $Z$ weifel $T y r^{2}$, der von den Südgermanen Mars Thingsus genannt wird ${ }^{3}$. In diesem Eid finden wir teils Spuren eines Gottesstaates, d.h. eines Rechtsstaates unter göttlicher Obhut, teils eines Götterstaates, oder wenigstens ein Fragment eines Pantheons. Waren die germanischen Gemeinwesen immer Gottesstaaten mit einem Götterstaat? Wir wollen diese Frage unter drei Hauptüberschriften behandeln.

\section{Die Existenz des Staates auf germanischem Boden}

Was ist ein Staat? Die wichtigsten Eigenschaften dieser Gemeinschaftsform ${ }^{4}$ werden von dem finnischen Westermarck-Schüler Rudolf Holsti so

1 Text in Den norsk-isländska skaldediktningen, rev. av E. A. Kock, т, Lund 1946, S. 60 und 96 .

2 So, wenn Ullr = Tyr, schon H. Pálsson, „Áss hinn almáttki“, Skirnir, I30, 1956, S. $187 \mathrm{ff}$. Gewöhnlich werden die Worte almáttki óss auf Odin oder Thor bezogen (siehe J. de Vries, Altgermanische Religionsgeschichte, 2, Berlin I957, S. I50: 2).

${ }^{3}$ G. Dumézil, Mythes et dieux des Germains, Paris 1939, S. 39 f.

4 Über diesen Begriff A. V. Ström, Religion och gemenskap. Studier $i$ religionssociologi, Uppsala 1946, S. 23, und G. Mensching, Soziologie der Religion, Bonn r968, passim. 
angegeben: Der Staat, is organized under the rule of a government which is independent of other governments, and it must also occupy a territory of its own"1.

Man hat Andeutungen von Staatsbildungen schon während der Steinzeit finden wollen. Leute, die Bohlenwege bis zu $25 \mathrm{~km} \mathrm{lang}$, aus zehntausenden von Stämmen bestehend, und Grabanlagen für mehrere Hundertzahl Personen haben bauen können, müssen, sagt man, ,ein größerer, staatlicher Verband" mit ,Staatsoberhäupter" ausgemacht haben². In der Steinzeit wissen wir aber nichts über eine Unabhängigkeit von anderen Machthabern oder über einen eigenen Bereich. Auch entbehren wir jede Kenntnis eines Zusammenhangs zwischen der eventuellen Organisation und der Religion, d. h. wir besitzen keine Kennzeichen eines Gottesstaates. Während der Bronzezeit dagegen gibt es möglicherweise Spuren von Staatsbildungen.

Erst hat J. Weisweiler gezeigt, daß sumer. pa-te-si, ,Priesterfürst', ,Stadtfürst',

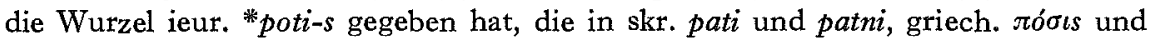
лótvia, lat. potestas, lit. pats und pati u. s. w. zum Vorschein kommt ${ }^{3}$. Daraus schließt dann H. Mitteis an das Dasein von ,größeren, straffer geleiteten Staaten“, die zu frühen Fürstengräbern Veranlassung gegeben haben sollen ${ }^{4}$. Reinhard Wenskus, der die Auseinandersetzung zusammengefaßt hat, zeigt u. a. auf das s. g. Königsgrab in Seddin, südlich von Potsdam, das ist ein 1o $\mathrm{m}$. hoher und $85 \mathrm{~m}$. breiter Grabhügel aus der Bronzezeit (jüngere Lausitzer Kultur) mit reichem Inhalt und Resten eines 30-40-jährigen Mannes und zwei junger Frauen ${ }^{5}$. Das Grab liegt im Bereich des später bezeugten Kultverbandes der Semnonen (Tac. Germ. Kap. 39$)^{6}$. Der alte idg. Wortstamm *rēg- könnte nach diesem Zeitabschnitt aus dem Germanischen

${ }^{1}$ R. Holsti, The Relation of War to the Origin of the State, Helsingfors I9I3, S. 5. Ähnlich W. F. Ogburn-M. F. Nimkoff, A Handbook of Sociology, London 1947, S. 4II: ,The state is a distinct and separate social institution with ultimate control over all other groups found within the given area." Vgl. R. M. MacIver, Society. $A$ Textbook of Sociology, New York I947, Kap. ,The State and the Greater Society“", S. 293 ff., und V. Aubert, Sosiologi, Kristiansand 1964, S. 118.

2 F. Genzmer, „,Staat und Gesellschaft", Germanische Altertumskunde, hrsg. von H. Schneider, München I95I, S. I47.

3 J. Weisweiler, „Das altorientalische Gottkönigtum und die Indogermanen“, Paideuma, 3, 1944, S. I 12 ff.

${ }^{4}$ H. Mitteis, Deutsche Rechtsgeschichte, München und Berlin 1952, S. I3.

$5 \mathrm{R}$. Wenskus, Stammesbildung und Verfassung. Das Werden der frühmittelalterlichen gentes, Köln-Graz I96r, S. 344 f., vgl. S. 308-314. J. Filip, Enzyklopädisches Handbuch zur Ur- und Friihgeschichte Europas, 2, Prag 1966, Sp. I 275 f.

- Wenskus, S. 345 . 
verschwunden sein, um nur das Wort ,Reich' (ahd. rìhi, got. reiks u. s. w. ${ }^{1}$ ) und den Namen Rigr in Rigsp. zu hinterlassen, die nicht aus dem Keltischen geliehen sein müssen ${ }^{2}$.

Von diesen Theorien abgesehen scheint man darüber ziemlich einig zu sein, daß wirkliche Staaten in unsrem Sinne nicht vor dem Übergang zwischen keltischer und römischer Eisenzeit, d. h. um die Geburt Christi, geschichtlich dargetan werden können. Dem zuvor können wir mit Sicherheit nur zwei größere Gemeinschaftsformen feststellen, nämlich die Sippe und die Altersklasse.

Von der Sippe ist nicht viel zu sagen. Die Familie war oft die Großfamilie $^{3}$ mit den verheirateten Söhnen im Elternhaus oder in dessen Nähe wohnend ${ }^{4}$. „Das germanische Altertum kennt im allgemeinen noch nicht die Kleinfamilie von heute", sagt Friedrich Stroh etwas übertrieben", aber wenn man archäologisch sehr große Bauernhäuser, bis zu $50 \mathrm{~m}$. Länge, entdeckt hat ${ }^{6}$, gewinnt man anschauliche Beispiele von der Umfassung der Großfamilie? ${ }^{7}$ Man kann weiter etwa 50 Personen in dem Hofe Bergthorshval rechnen, Njal und Bergthora, deren drei Söhne und eine Tochter, Njals illegitiemen Sohn, nebst Schwiegertöchtern, Schwiegersohn und Enkeln ${ }^{8}$.

${ }^{1}$ F. Kluge, Etymologisches Wörterbuch der deutschen Sprache, bearb. von W. Mitzka, Berlin r967, S. $59 \mathrm{r}$.

a Wenskus, S. 357 (vgl. jedoch S. 359 !). Die traditionelle Entlehnungshypothese bei J. de Vries, Altnordisches etymologisches Wörterbuch, Leiden I963, S. 80.

3 Über diesen Begriff und die entsprechenden Englischen siehe T. N. Madan, „The joint family: a terminological clarification", Family and Marriage, ed. by J. Mogey, I $_{93}$, S. 7 ff. „,All property is held in common" in der Großfamilie, und die Mitglieder ,participate in common worship" (S. I I).

"Siehe S. Erixon, „Gảrden och familjen“, Etnologiska studier tillägnade N. E. Hammarstedt, I921, S. 195 ff., M. Olsen, AEttegård og helligdom, Oslo 1926, S. 3 I ff., A. O. Johnsen, Fra ættesamfunn til statssamfunn, Oslo I948, S. 6 I f., 68 ff., Mensching S. 34 ff.

${ }^{5}$ F. Stroh, Handbuch der germanischen Philologie, Berlin 1952, S. I79. Vgl. H. Begemann, Strukturwandel der Familie, Witten I966, S. 26, und Johnsen, S. 61 und 68.

6 Beispiel: der Bauernhof Bø auf Jæren in Norwegen (Olsen, S. 39).

? Verwarnungen gegen unbedachtes Benutzen des Wortes Sippengesellschaft spricht Hellström aus (J. A. Hellström, Biskop och landskapssamhälle $i$ tidig svensk medeltid (Skrifter utg. av Institutet för rättshistorisk forskning, I: I6), Motala 1971, S. I3).

${ }^{8}$ Njála Kap. $25-27$ und 127 ( $I F$ I2), vgl. Vatsd. Kap. 27 (IF 8) und Gautr. Kap. 2. 
Die Gesetze verbieten, noch in christlicher Zeit, das Begraben in den Hügel anderer Sippen (grafua a æetce högh annars)'.

Um so mehr sollte man der Entwicklung Altersklasse - Männerbund Gefolgschaft, welche die Entfaltung des germanischen Staates wiederspiegelt, Aufmerksamkeit schenken, da diese Linie noch nicht gebührend beachtet worden ist.

Nach Vorarbeiten von Leo Frobenius in den goer Jahren wurden die Altersklassen und Männerbünde von Heinrich Schurtz I902 zum ersten Male beschrieben $^{2}$ und dann von $H$. Webster, S. Luria, B. Favre und $E$. Hildebrand in verschiedenen Kulturen studier ${ }^{3}$. Auf germanischem Boden wurden Untersuchungen von Sigurd Erixon I92I, Lily Weiser-Aall 1927 und vor allen Otto Höfler I 934 ausgeführt ${ }^{4}$.

Im Jahre 1938 erschien so Stig Wikanders bahnbrechendes Buch, das das Vorkommen kriegerischer Männerbünde unter den Indiern und Iraniern nachgewiesen hat, die durch militarische Organisation, ekstatische Kampfweise, freie Liebesverhältnisse und geheimen Kult ringsum einen Heros und Drachentöter gekenntzeichnet waren ${ }^{5}$. Imselben Jahr ergänzte Geo Widengren unsre Kenntnis der Män nerbünde durch Analyse iranischer Texte ${ }^{6}$, und im folgenden Jahr erschienen teils Richard von Kienles Ausführungen über den Bund und den Zusammenhang zwischen den Männerbünden und der Gefolgschaft des Fürsten', teils Georges

${ }^{1}$ Smålandslagen (Smolandisches Gesetz) § I7 (CISA, 6, I844), S. I Io, vgl. $N G L$, I, I 846 , S. $4 \circ 5$.

2 L. Frobenius, Die Geheimbünde Afrikas, Hamburg 1894 ; L. Frobenius, Die Masken und Geheimbïnde Afrikas, Halle 1899; H. Schurtz, Altersklassen und Männerbünde, Berlin I902.

${ }^{3}$ Die Literatur in Ström, Religion och gemenskap, S. roo. Später: J. Haekel, "Initiationen und Geheimbünde an der Nordwestküste Nordamerikas", Mitteilungen der Anthropologischen Gesellschaft in Wien, 83, 1954, S. 167 ff.; B. Lindskog, African Leopard Men (Studia Ethnographica Upsaliensia, 7), Uppsala r954.

"S. Erixon, „Ynglingalaget", Fataburen, I92I, S. 95 ff.; Lily Weiser[-Aall], Altgermanische füinglingsweihen und Männerbïnde (Bausteine zur Volkskunde und Religionswissenschaft, I), Bühl 1927; O. Höfler, Kultische Geheimbiinde der Germanen, I, Frankfurt am Main I934, dessen 2. (von R. Stumpf, Kultspiele der Germanen als Ursprung des mittelalterlichen Dramas, Berlin 1936, S. $39 \mathrm{I}$ ff. referiertes) Band leider noch nicht erschienen ist; A. Slawik, ,Kultische Geheimbünde der Japaner und Germanen", Die Indogermanen- und Germanenfrage (Wiener Beiträge zur Kulturgeschichte und Linguistik, 4), I936, S. 675 ff.

5 S. Wikander, Der arische Männerbund. Studien zur indo-iranischen Sprach- und Religionsgeschichte, Lund I938, S. 64 ff., vgl. S. Wikander, Vayu. Terte und Untersuchungen zur indo-iranischen Religionsgeschichte, I (Quaestiones indo-iranicae, $\mathrm{r}$ ), Lund I 941 , S. $128 \mathrm{ff}$.

${ }^{6} \mathrm{G}$. Widengren, Hochgottglaube im alten Iran. Fine religionsphänomenologische Untersungung (Uppsala universitets årsskrift, 1938: 6), Lund 1938, S. $320 \mathrm{ff}$.

7 R. von Kienle, Germanische Gemeinschaftsformen (Deutsches Ahnenerbe, Reihe B, Abt.: Arbeiten zur Germanenkunde, 4), Stuttgart 1939, S. 137 ff. 
Dumézils besser soziologisch orientierte Darstellung der germanischen Schreckkrieger ${ }^{1}$. Jan de Vries hat 1943 über Sippe und Gefolgschaft als Wurzeln des germanischen Staatswesens geschrieben ${ }^{2}$, und vor kurzem hat Widengren im einzelnen gezeigt, daß die germanische Gefolgschaft bezüglich militarischer und religiöser Organisation und Sitte genaue idg. Parallele in Griechenland, Rom, Iran und Indien hatte ${ }^{3}$.

Tacitus bezeugt, daß die Sippe und die Gefolgschaft die Wurzeln des Staates auf germanischem Boden sind. Er spricht von dem Staat (civitas, Germ. I0: I, I3: I, respublica, I3: I) und setzt Staatsämter voraus: Könige werden ausersehen aber ,mit Bezug auf hohe Geburt" (ex nobilitate), Heerführer dagegen, ,infolge Tapferkeit", oder eher ,Tüchtigkeit" (ex virtute, $7:$ I). Bald darauf wird aber erzählt, eine Reiterabteilung (turma) oder ein Keil (cuneus) bestehe aus Familien (familiae) und Geschlechter (propinquitates, 7:2). Noch zur Zeit Caesars waren die Fürsten auch Stammälteste ${ }^{4}$, und so spät wie im I 1 . und 12 . Jahrhundert waren die Vasallen in Norwegen ,mit den Königen durch nahe Verwandtschaftsbande verbunden"s. Tacitus kennt aber auch die Begriffe Gefolgschaft (comitatus), Gefolgsleuten (comites) und Gefolgsherr (is quem sectantur, 13:2) ${ }^{6}$, und wir kennen die entsprechenden germanischen Bezeichnungen: teils lid, Gefolge $^{6}$, teils piakn>pegn und hempegi $>$ heimpegi $i^{8}$.

' Dumézil, Mythes et dieux des Germains, S. 97 ff. (les guerriers-fauves), vgl. G. Dumézil, Horace et les Curiaces, Paris 1942, S. $16 \mathrm{ff}$.

2 J. de Vries, Die geistige Welt der Germanen (Handbiicherei der Deutschkunde, 7), Halle/Saale I943, S. $40 \mathrm{ff}$. (Verwandtschaft), S. $57 \mathrm{ff}$. (Gefolgschaft), S. $63 \mathrm{ff}$. (Staat). Vgl. H. Conrad, Deutsche Rechtsgeschichte, I, Karlsruhe 1954, S. 23 ff.

a G. Widengren, Der Feudalismus in alten Iran. Männerbund - Gefolgswesen Feudalismus in der iranischen Gesellshaft im Hinblick auf die indogermanischen Verhältnisse (Wissenschaftliche Abhandlungen der Arbeitsgemeinschaft für Forschung des Landes Nordrhein-Westfalen, 40), Köln und Opladen 1969, S. $47 \mathrm{ff} ., 96 \mathrm{ff}$.

${ }_{4}^{4}$ W. Schulz, Staat und Gesellschaft in germanischer Vorzeit (Vorzeit. Nachweise und Zusammenfassungen zu dem Arbeitsgebiet der Vorgeschichtsforschung, hrsg. von H. Hahne, 4), Leipzig I926, S. I3, vgl. Wenskus, S. $325 \mathrm{ff}$.

5 H. Koht, ,Hvem var Tore Ingeridson?“, [Norsk] Historisk Tidsskrift, 26 [5: 5], I 924 , S. I4I.

6 Über die Gefolgschaft bei Tacitus siehe von Kienle, S. I44ff.; Widengren, Der Feudalismus, S. $47 \mathrm{f}$.

7 z. B. auf dem Glavendrup-Stein, Lis Jacobsen-E. Moltke, Danmarks Runeindskrifter. Lommeudgave, København I942, S. 57.

\& S. Bugge-M. Olsen, Norges Indskrifter med de ældre runer, 2: I, Christiania I917, S. 563; E. Wessén-S. B. F. Jansson, Upplands runinskrifter, 2, Uppsala 1946, S. I3 8 ff., und dazu de Vries, Die geistige Welt, S. 63; Widengren, Der Feudalismus, S. 53 . 
Über das Aufnehmen der Jünglinge in diese Klasse schreibt Tacitus:

Ante hoc domus pars videntur, mox rei publicae.
Zuvor werden sie als ein Teil der Familie angesehen, dann einer des Staates.

(Tac. Germ. Kap. 13: I)

In Kap. 7 wird aber über die Rolle der Religion und der Priester im Staat gesprochen, und bald darauf wird sogar einen Staatspriester (sacerdos civitatis, то: I) erwähnt. Es kann also schon hier von einem Gottesstaat die Rede sein.

\section{Der Sinn des Gottesstaates}

Der Staat ist aus Geschlecht und Sippe entstanden, die für die Fruchtbarkeit der Erde und der Haustiere und für ihren eigenen Erfolg und Nachwuchs gesorgt haben, und aus Gefolgschaft und Heer, die sich mit kriegerischen Dingen beschäftigt haben. Und nun finden wir schon bei Tacitus einen Kult zugunsten der Götter eben dieser beiden Funktionen:

A. Ein Kult, dessen genaue Entsprechung wir später im Norden vorfinden, wird von einer ganzen Reihe von Stämmen, u. a. den Angliern, der Fruchtbarkeitsgöttin Nerthus, id est Terra mater (40:2) gewidmet. Geber der Zeugungskraft waren auch die dioskurischen Gestalten, die nach Tacitus, der in solchen Sachen sich nicht zu irren pflegt, und nach seinen modernsten Auslegern von Germanen verehrt wurden ${ }^{1}$, und deren Namen Tacitus wahrscheinlich ganz richtig als nom. alcis< germ. *alhiz, die Beschützenden, Helfenden' (wie die indischen aśvināu dhişnyāu) wiedergibt.

B. Ein kriegerischer Kult liegt auch vor. Für das ganze germanische Gebiet teilt Tacitus mit, der Familienkult und das Orakelwesen werde vom Familienvater besorgt (ipse pater familiae precatus deos, I0: I), was aber der Staatspriester (sacerdos civitatis) tut, gelte publice, ,auf die Rechnung des Staates' ( $i b$.) und beziehe sich auf das Kriegswesen (Io: 3). Hier erfahren wir die Namen der Götter nicht, aber man dürfte sicher auf die im vorigen Tacitus-Kapitel erwähnten Hercules und Mars (9: I) raten können.

Auch anderswo findet man Belege für das Dasein eines Gottesstaates. Durch vergleichende literarische und archäologische Forschung stellt Karl

1 Tac. Germ. 43: 3 und dazu Publius Cornelius Tacitus Germania, hrsg., übers. und mit Erläuterungen versehen von E. Fehrle. Aufl. besorgt von R. Hünnerkopf, Heidelberg 1959, S. 128 . Anders H. Biezais, „Die vermeintlichen germanischen Zwillingsgötter", Temenos, 5, 4969, S. 30 . 
Hauck fest, die in Sutton Hoo und auf anderen Fundplätzen zum Vorschein kommenden signa sacra seien "Zeichen des Herrschens und Hegens auf den Thing-, Kult- und Kampfplätzen der vorchristlichen Staatlichkeit1,“ ja, auf Grund der germanischen Stammesgenealogien könne die Folgerung gezogen werden, die staatliche Religion und Kultübung sei „eines der Hauptbindemittel dieser Staatlichkeit"“2.

Man kann aber vom Anfang an zwei parallel vorkommende Staatsverfassungen unter den Germanen, unseren Termen Republik und Monarchie entsprechend, unterscheiden: Staatsformen ohne Könige oder mit Königen ${ }^{3}$. Allen beiden zugrunde lag aber das Recht, das ganz und gar religiös begründet war. Hier kann an einer bezeichnenden Tatsache angeknüpft werden: obwohl ein germanisches Gericht sich keineswegs die Aufgabe stellte, in erster Linie die Wahrheit zu finden, sondern die formale Richtigkeit des Eides festzustellen (vgl. z. B. Glüma Kap. 25), war die Kraft des Eides ganz von dem Glauben an die Götter abhängig, denn ein falscher Eid forderte den Zorn der Götter heraus".

Sowohl die rechtspflegende als auch die regierende Macht lag bei dem Ding (pingr), dessen Funktionen vor kurzem von Jan Arvid Hellström untersucht worden sind ${ }^{5}$. Diese waren nicht nur rechtssprechend sondern galten auch dem Heerbannswesen (leiđangr), der Besteuerung und sowohl politischen als auch verwaltungsmäßigen Fragen ${ }^{6}$. Über die kultische Funktion des Dinges äußert sich Hellström unschlüssiger aber erwähnt die meiner Meinung nach entscheidenden Faktoren?: Kultakten und Götterbilder an den Dingplätzen, sakrale Urteile, Dingsfrieden, Dingsunverletzlichkeit und die Eidsformulare, vor allem das im älteren Westgötagesetz zwanzigmal wiederkehrende:

${ }^{1}$ K. Hauck, „Herrschaftszeichen eines Wodanistischen Königtums", Festgabe Anton Ernstberger (Gahrbuch für fränkische Landesforschung, I4), 1954, S. 58.

2 K. Hauck, ,Lebensnormen und Kultmythen in germanischen Stammes- und Herrschergenealogien", Saeculum, 6, I955, S. I 86.

${ }^{3}$ Genzmer, S. 148 . Über dieses Gegensatzpaar sagt Wenskus: „Dieser neuzeitlichen Antithese steht im Altertum die viel angemessenere, wenn auch gleichfalls ungenügende Trias Monarchie: Aristokratie: Demokratie gegenüber" (S. 3 I 3 f.).

${ }^{4}$ Genzmer, S. I29. - Alle Götter als Zeugen zu nehmen steht in Gísla, Kap. 6.

5 Hellström, S. I 9 ff.

' Hellström, S. I20.

7 Hellström, S. I I9, I25 und 155. 
bipiæ sva sær gud holl ok uattum sinum $\mathbf{1}$. den Göttern zu beten, sich und seinen Zeugen gnädig zu sein.

Besonders zu beachten ist, meine ich, daß Kultgehörigkeit und Dingzutritt von Tacitus zusammen genannt werden:

nec aut sacris adesse

aut concilium inire ig-

nominioso fas.
Der Ehrenlose darf weder

dem Gottesdienst beiwohnen noch auf dem Ding auftreten.

(Tac. Germ. Kap. 6: 4)

Die am Ding ausgeübten Staatsfunktionen enthielten lange viel von der Sippenideologie. Die Zeugen waren zuerst Verwandte, und der Sippe überließ man, auch im späteren staatlichen Strafrecht, die Bestrafung, wenn Angehörige an der Ehre gekränkt worden waren ${ }^{2}$. Eine interessante Beobachtung hat Bertha Phillpotts schon lange her gemacht: wo das Sippengefühl stark war, wie in Dänemark und Südschweden, war auch die Freiheit und die Demokratie größer, aber wo das Verwandtschaftsgefühl durch ,migration by sea" geschwächert worden war, wie in England, Norwegen und Island, wurde die Freiheit vermindert ${ }^{3}$.

Die zweite germanische Staatsform war das Königtum. Der sakrale König vertrat einerseits das Volk vor dem Gott und andererseits den Gott vor dem Volk ${ }^{4}$. Das germ. Wort *kuninga-z>konungr hängt einigermaßen mit konr, ,Sohn, Mann'<*kunja, ,Sippe, Geschlecht', zusammen ${ }^{5}$. Richard Ekblom hat darin einen ,Mann eines Geschlechts', ,Ablömmling', gesehen', Jan

1 ,Giptar bolker" § 9: 5, CISA, I, I827, S. 36. Vgl. Svenska landskapslagar, tolkade och förklarade för nutidens svenskar av A. Holmbäck och E. Wessén, 5, Uppsala 1946, S. I6: 41. Die entsprechende christliche Formel: sva se mer gud hollr in „Hirdskraa“ § 10, NGL, 2, I 848 , S. 398.

${ }^{2}$ Genzmer, S. I32.

${ }^{3}$ Bertha S. Phillpotts, Kindred and Clan in the Middle Ages and After. A Study in the Sociology of the Teutonic Races, Cambridge 1913, S. $254 \mathrm{f}$.

4 O. Höfler, „Das Opfer im Semnonenhain und die Edda", Edda, Skalden, Saga. Festschrift für Felix Genzmer, I 952, S. I4 f.; A. V. Ström, ,"The King God and his Connection with Sacrifice in Old Norse Religion", RegSacr., S. $703 \mathrm{ff}$. und Wenskus, S. 314.

${ }^{5}$ de Vries, Altnordisches etymologisches Wörterbuch, S. 326; Kluge, Etymologisches Wörterbuch, S. 392.

6 R. Ekblom, ,Germ. *kuningaz 'König'“, Studia neophilologica, I7, 1944-45, S. 4. Diese Deutung wird von H.-D. Kahl, „Europäische Wortschatzbewegungen

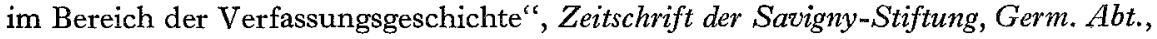
77 , r960, S. r63: 2 I, bestritten. 
Gonda und G. Dumézil gleicherweise ,den Repräsentant des Volkes'1. F. R. Schröder führt das Wort auf *kunjaz = lat. genius, ,Erzeuger', zurück, also ,Nachkomme des Erzeugergottes' oder ,Partner der Muttergöttin', während de Vries es direkt aus konr ableitet: 'Mann von göttlicher Abkunft'3. Bei allen diesen Deutungen liegt die Vorstellung von Verwandtschaft und Zeugung dahinter.

Wichtiger sind die Wurzeln des Königtums. Gemäß Tacitus besaßen die Priester allein die wirkliche Macht sowohl im Krieg als auch im Frieden

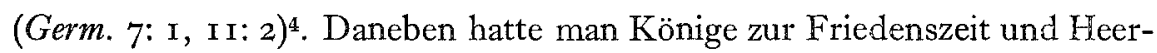
führer (ahd. hęrizoho > Herzog) in der Streit (Caesar, De bell. Gall. VI,23, Tac. Germ. 7: I). Die Strafen aber, sagt Tacitus, werden verteilt

non quasi in poenam nec ducis iussu, sed velut deo imperante. nicht als Bestrafung oder auf $\mathrm{Zu}$ sage des Herzogs, sondern gleichwie auf Befehl des Gottes.

(Tac. Germ. 7: 1).

Der Staat ist also ein Gottesstaat.

Schon Schulz hat klar gesehen, daß das Königtum in geschichtlicher Zeit , aus der Herzogwürde und aus der Priesterwürde hervorgegangen ist“"5. Das Priestertum dürfte das älteste Amt sein. In nordischen Fürstengräbern aus dem Ende der Bronzezeit ist nicht viel Schmuck und Prunk gefunden worden, aber statt dessen kultische Gegenstände. „Hier scheint eine geistigpriesterliche Komponente vorzuwiegen," sagt Wenskus auf Grund der archäologischen Forschungen von E. Wahle, der seinerseits in dem Grabinhalt „,noch ein Erbgut aus der Zeit des indogermanischen Urfolkes“ sieht ${ }^{6}$. Später tritt die adelig-militarische Komponente ein. Das soziologische Lehrgedicht Rigspula, das über die drei indogermanischen Volksschichten Bescheid gibt, beschreibt wie Konr ungr ('der junge Mann' = konungr,

1 J. Gonda, ,Semantisches zu idg. rēg- 'König' und zur Wurzel reg- ('sich ausstrecken'), Zeitschrift für die vergleichende Sprachwissenschaft, 17, 1956, S. 166; G. Dumézil, Mitra-Varuna, Paris 1948, S. 63.

2 F. R. Schröder, Ingunar-Freyr (Untersuchungen zur germanischen und vergleichenden Religionsgeschichte, I), Tübingen I94. I, S. 33 ff.

${ }^{8} \mathrm{~J}$. de Vries, ,Das Königtum bei den Germanen", Saeculum, 7, 1956, S. $298 \mathrm{ff}$.

${ }^{4}$ Schultz, S. ${ }_{4}$.

$5 \mathrm{Ib}$.

${ }^{8}$ E. Wahle, Deutsche Vorzeit, Tübingen 1952 , S. 137 f.; Wenskus, S. $309 \mathrm{f}$. 
,der König') von Jarl und Erna (NB diesmal ohne Mitwirkung von Rigr < reĝ-!) geboren wird (Rigsp. $4 \mathrm{I}$ f.) ${ }^{1}$.

Betreffs des Königwerdens hat Widengren gezeigt, daß man auf indoiranischem Boden eine charakteristische Zwischenform zwischen Erb- und Wahlreich gekannt hat, und diese spezifische idg. Form haben Dänen, Svear, Götar, deutsche Stämme, Iren und vielleicht Galliern gepflegt, während nur Norwegen ein reines Erbesystem gehabt hat ${ }^{2}$. Das Wort Wahlreich ist für germanische Verhältnisse nicht ganz passend. Schon Tacitus weiß, daß die Könige in Germanien nicht gewählt sondern ,genommen' wurden (sumere statt eligere, Germ. 7: I). Der, angenommene, ausersehene' König sollte dann von den ,Gesetzsprechern' (logsegumenn = Vorsitzenden) der verschiedenen Gesetzdingen (log ping) ,zum König verurteilt = bestellt werden" (döma til konung) ${ }^{3}$. Die Wörter Wahl und Wählen (electio, eligere) waren in vorchristlicher Zeit den Satzungen über Besetzen des Königsamts fremd, und erst die Kirche hat diese Begriffe in die Gesetze hineingeführt ${ }^{4}$.

Der „,zum König verurteilte“ besaß keine unbeschränkte Macht (vgl. oben S. 147). Dies bezeugt Tacitus für die römische Eisenzeit, Adam von Bremen und Snorri Sturluson für die Wikingerzeit:

nec regibus infinita

aut libera potestas ${ }^{5}$.

Reges habent ... quorum vis pendet in populi sententia.

Svá hafa gert allir Svia-konungar, at láta boendr ráda med sér qllu poi er peir vildu.
Die Könige haben keine unbeschränkte oder freie Macht. (Tac. Germ. Kap. 7: I).

Sie haben Könige ... deren Macht im Willen des Volkes liegt. (Adam, Gest. Hamm. IV, 22). So haben alle Svear-Könige getan, $\mathrm{da} ß$ sie die Bauern mit sich in allen Dingen raten lassen, wo sie wollten. (Snorri, Ól. Helg. Kap. 8o) ${ }^{6}$.

${ }^{1}$ Dazu G. Dumézil, „La Rígspula et la structure sociale indo-européenne“ $R H R$, I 54, I958, S. I ff.

2 Widengren, Der Feudalismus, S. 148, nach G. Dumézil, Servius et la Fortune, Paris I943, S. 22, If. Widengren faßt Götar und Svear als ,Schweden“ zusammen.

${ }^{3} \mathrm{~K}$. Olivecrona, Das Werden eines Königs nach altschwedischem Recht (Lunds universitets årsskrift, N.F. I, 44: I), Lund I947; G. Hafström, „Konge, Sverige“, Kulturhistoriskt lexikon för nordisk medeltid, 9, 1964, Sp. го f.

* Ib.; Hellström, S. I64.

` Vgl, über die Gothen: nondum tamen supra libertatem (Tac. Germ. 44: I).

- Heimskringla, Nóregs konunga sogur af Snorri Sturluson, udg. ved F. Jónsson (Samfund til Udgivelse af gammel nordisk Litteratur, 23), København I899, S. 144. 
Hellström faßt zusammen: „Zwischen König und Ding bestand ein deutliches Zusammenspiel, nicht in erster Linie ein Gegensatzverhältnis"1. Ein Ausdruck dafür ist, meine ich, die alten stabreimenden Worte

karl eller konunger

Mannschaft oder König

(Smål. Gesetz, Kirchenrecht, Kap. 2). ${ }^{2}$

Otto Höfler hat ausführlich dargelegt, daß die germanischen Könige, weil das Volk selbst als göttlichen Ursprungs galt, „nicht als substantiell vom Volk geschieden angesehen wurden, sondern als primi inter pares"3. In dem germanischen Staat waltete also "die ursprüngliche Demokratie“ (primitive democracy) derselben Art, wie die die man unter den Sumerern gefunden hat ${ }^{4}$. Das Königtum war nichts über das Volk Erhabenes, es war ,gesteigerter Adel“5. Andererseits begegnen wir einem interessanten Verhältnis: wo die herrschende Sippe ausstirbt, scheint das Schicksal des Stammes besiegelt zu sein, wie die Beispiele der Cherusker, Markomannen, Goten, Wandalen u. a. zeigen ${ }^{6}$.

Die Organisation des Staates fußte anfangs der Gefolgschaft, dem persönlichen Kreis um den König. Diese Gemeinschaft war nämlich deutlich auf Rangunterschieden aufgebaut (Tac. Germ. Kap. 13), vor allem hinsichtlich der Geburt und des Ansehens?. Viele verwaltungsrechtliche Begriffe zeigen das Persönliche beim germanischen Staat, z. B. die Wörter für ,Bezirk': norw. fylki<folk, ,Volk', schwed. haera $p>$ härad und dän. haeraeth $>$ herred $<{ }^{*}$ harja-raido, , reitende Schar' $=$ die Gefolgschaft ${ }^{8}$. Erst der römische Einfluß schuf Ermanarichs westgotisches, Theoderichs ostgotisches und Chlodowechs fränkisches Reich mit ihrem amtlichen Apparat ${ }^{9}$.

${ }^{1}$ Hellström, S. I22 (meine Übersetzung).

2 „Kristnu-balken" $\$ 2, C I S A, 6$, I844, S. 97.

s O. Höfler, „Der Sakralcharakter des germanischen Königtums", RegSacr., S. 695 .

4 Siehe A. Haldar, oben S. $3^{\text {I. [Red.] }}$

5 K. Hauck, „Die geschichtliche Bedeutung der germanischen Auffassung von König und Adel", $I I^{e}$ Congrès International des Sciences Historiques Stockholm, 2I-28 août I960, Rapports 3, Moyen âge, I96o, S. IоI.

- Wenskus, S. $66 \mathrm{ff}$.

7 Wenskus, S. 329 f.

${ }^{8}$ de Vries, Alnordisches etymologisches Wörterbuch, S. 148; E. Hellquist, Svensk etymologisk ordbok, I, Lund r97o, S. $3^{87}$. Vgl. von Kienle, S. 269; Wenskus, S. 69.

${ }^{9}$ de Vries, Die geistige Welt der Germanen, S. 65. 
Jedoch heißt es nimmer z. B. rex Franciae sondern rex Francorum, Vandalorum u. s. w. ${ }^{1}$, und Missionare wurden nicht nach Schweden, Dänemark oder Polen gesandt aber

gentibus Sueonum sive Danorum

zu den Völkern der Schweden, der Dänen nec non eitiam Slavorum. ${ }^{2}$ und auch der Slaven.

Der germanische Staat war, um mit Buccellati zu sprechen, kein Territorialstaat sondern ein Nationalstaat ${ }^{3}$. Außerdem knüpften die Könige der nordischen Länder des 9. Jahrhunderts, „die ersten Königreiche die in zuverlässigen Quellen erwähnt sind“, stark an die Städte und deren Handel an ${ }^{4}$.

Zusammenfassend läßt sich sagen, daß die Fundamente des Gottesstaates in Germanien dreifacher Art sind:

I. die Autorität in zwei Formen: a. regierend-königlich

b. juristisch-priesterlich

2. die Kampfbereitschaft der Gefolgschaft

3. die Fruchtbarkeit der Sippe und des Volkes.

Das sind gerade die Funktionen, die in dem alten idg. Götterstaat tätig sind, und wir gehen dazu über.

\section{Das Auftreten des germanischen Götterstaates}

In der vedischen Theologie begegnen wir einem Götterstaat mit Varuna als samrāj, ,Allkönig', oder Mitrāvarunā als rājānau, ,Könige', und retasya gopau, ,Behüter der Ordnung', weiter mit Indra als Vrtrahan, 'Drachentöter', und mit Aśvinau als den helfenden Fruchtbarkeitsgöttern (z. B. Sat. Br. IV, I: $3-5)^{5}$. In Rom sind die Götter gleichermaßen unter Führung von fuppiter O.M. gesammelt ${ }^{6}$. Unter den Germanen aber stehen die Sachen

1 de Vries, Die geistige Welt der Germanen, S. 66; Wenskus, S. 7т.

${ }_{2}$ Sveriges traktater med främmande magter jemte andra dit hörande handlingar, utg. af O. S. Rydberg, I, Stockholm 1877, S. 3 f. Dazu Hellström, S. I4 f., vgl. S. 34 .

3 Siehe B. Albrektson, oben S. 46. [Red.]

${ }^{4}$ E. Lönnroth, Acta Visbyensia, I, Uppsala 1965, S. I7.

${ }^{5} \mathrm{G}$. Dumézil, L'idéologie tripartie des Indo-Européens (Collection Latomus, $3 \mathrm{I}$ ), Bruxelles I 958, S. 34 und $3^{8}$ f.; A. V. Ström, „,Die Inder der klassischen Zeit" und „Die Germanen", H. Ringgren- $\AA$. V. Ström, Die Religionen der Völker. Grundriß der allgemeinen Religionsgeschichte (Kröners Taschenausgabe, 291), 1959, S. $188 \mathrm{ff}$. und $39 \circ \mathrm{ff}$., besonders S. 194 .

' Dumézil, L'idéologie tripartie, S. 48 f. 
etwas anders. Man kann sogar, mit de Vries, von einem „schwachen Gemeinschaftsgefühl" in bezug auf Volk und Staat sprechen. Die Empfindung für Familie, Sippe, Blutbruderschaft, Gefolgschaft und die Verbindung Erzieher -Erzogener ist reichlich entwickelt ${ }^{2}$, aber ,über der Gesichtskreis der Sippe blickt der Germane nur selten hinaus“"3. So war auch die Frömmigkeit merkwürdig individuell oder an das Geschlecht gebunden: man wählt sich einen Gott unter den Vielen als seinen fulltruii, ,Vertrauten', ,Privatgott', (Eir. raud. Kap. 7, Porl. páttr jarlask., vgl. Eyrb. Kap. 3, Hrafnk. Kap. 3). Weiter kann man sich von einem Gott zum anderen umkehren (Gluma Kap. I9, 23 und 26), und ein Geschlecht verehrt einen besonderen Gott (Lnb. H. Kap. I84, Gluma Kap. I). Der Wiking ruft Thor an, der Bauer opfert zu Frey, Jarle leiten ihren Stammbaum von Odin her, die Familie sammelt sich um Freyja und die Disen u. s. w. Allmählich ist aber auch unter den Germanen das alte idg. Muster, im zurückgeschobenen aber nimmer vergessenen, Götterstaat hervorgetreten (Snorri, Gylf. Kap. 20).

Meine These ist jetzt, daß das Hervorwachsen des Gottesstaates die Voraussetzungen für das Wachrufen des Mythus vom Götterstaat geschaffen hat. Die Merowingerzeit auf dem Kontinent und die Wikingerzeit im Norden verehren einen Götterstaat reinster indogermanischer Art. Drei Erneuerungen bestätigen das.

\section{A. Die Stellung Odins.}

Unlängst hat man viel über eine Einwanderung Odins gesprochen", und noch kommt es vor, daß man es als ,wohlbegründet" bezeichnet, "Odin als eine relativ späte Gottheit unter den germanischen Völkern zu betrachten“, ja, man „stellt sich vor, daß der Kult für diesen Gott sich vom Südosten her auf die übrigen Teile der germanischen Welt kurz vor oder während der Völkerwanderungszeit verbreitet habe“" Darum dreht es sich aber gar

${ }^{1}$ de Vries, Die geistige Welt der Germanen, S. 65.

2 de Vries, Die geistige Welt der Germanen, S. $40 \mathrm{ff}$.

3 de Vries, Die geistige Welt der Germanen, S. 64.

${ }^{4}$ Am spätesten vielleicht K. Helm, Wodan. Ausbreitung und Wanderung seines Kultes, Amsterdam I968, S. 6 f.

${ }^{5}$ L. Ejerfeldt, „Germanische Religion", Handbuch der Religionsgeschichte, hrsg. von J. P. Asmussen und J. Laessøe, I, I97I, S. 288. 
nicht, besonders weil die dahinterliegende Erklärung der Ortsnamen sich als unzulässig erwiesen hat ${ }^{1}$, sondern um die neue Stellung Odins als Oberhaupt der Götterstaat. Und dem können wir beipflichten: „Ođđinn must have aquired his position as head of an organized system of gods in comparatively recent times".

Diese neue Stellung Odins in der Götterwelt wird nun mit Ausdrücken aus der Ideologie des Gottesstaates auf Erden geschildert ${ }^{3}$. Snorri betont vielerwärts das Primat Odins (Gylf. Kap. 9 und 20), vor allem in der Ynglingasaga Kap. 6-9, wo er Odin in der Spitze von 12 Gefolgsleuten (Kap. 7) als gesetzgebenden und besteuernden König (Kap. 8) darstellt. Bei Widukind von Corvey wird Wodan pater patrum genannt (I, II), und in Vita Kentigerni wird Woden principalis deus Anglorum geheißen (Acta Sanct. I, S. 820$)^{4}$.

Auch in der vorchristlichen nordischen Skaldendichtung gibt es deutliche Spuren von dem Götterstaat. Egill Skallagrimsson dichtet einen Schimpfvers $($ nid):

Reiđ̈ sé rogn ok Óđinn!

...

Folkmýgi làt floeja,

Freyr ok Njorär, af jorđum!

Leiđisk lofäa striḋi

landọss...
Zornig seien die Waltenden [Götter] und Odin! ...

Laß den Volksplager, Frey und Njord, von der Erde fliehen!

Der Landase [Thor] sei des Männerfeindes überdrüssig ...

(Egill, Lausav. Str. 19) ${ }^{5}$.

${ }^{1}$ L. Ejerfeldt, „Germanische Religion“, Handbuch der Religionsgeschichte, hrsg. von J.P. Asmussen und J. Laessø, I, I97I, S. 288.

2 ,Des études récentes ont montré sufficamment que l'hypothèse ... est arbitraire et fallacieuse" (J. de Vries, "La toponymie et l'histoire des religions", $R H R$, I 45, I954, S. 214; A. V. Ström, „Tradition und Tendenz. Zur Frage des christlichvorchristlichen Synkretismus in der nordgermanischen Literatur", Syncretism, ed. by S. S. Hartman (Scripta Instituti Donneriani Aboensis, 3), I969, S. 242 ff.

3 P. Buchholz, „Perspectives for Historical Research in Germanic Religion“, History of Religions, 8: 2, I968, S. I32.

4 Diese These entspricht Dumézils Idee von der Entsprechung zwischen den soziologischen Verhältnissen und der Götterwelt in indogermansicher Zeit (L'idéologie tripartie, S. 7 ff., 34 ff.).

5 Hauck, „Herrschaftszeichen“, S. 32 ff.; Hauck, „Lebensnormen“, S. 2 I6 f.; K. Hauck, Goldbrakteaten aus Sievern. Spätantike Amulett-Bilder der 'Dania Saxonica' und die Sachsen-'Origo' bei Widukind von Corvey (Mïnsterische MittelalterSchriften, I) München 1970, S. 44 (vg1. S. 232: 642). 
Gleich wie in der Ulfljot-Gesetz-Formel (oben S. I43) begegnet uns hier ein Pantheon aber jetzt mit den Göttern der drei idg. Funktionen und mit Odin an der Spitze.

\section{B. Der Charakter Odins.}

Dieser hat sich in dieser Verbindung etwas verschoben. Odin ist jetzt nicht nur der Typus, der als „,cosmique, magicien, terrible“ beschrieben wird ${ }^{1}$, sondern ist der Gott der Könige, des Staates und des Krieges durch „,un gauchissement", eine Linksschiebung, ein Übernehmen gewisser Mustern der zweiten Funktion ${ }^{2}$.

Eine Folge ist, daß wir Odin-benannte Plätze fast in der ganzen germanischen Welt finden, z. B.:

Deutschland: Wudenesberg $>$ Godesberg

Holland: Woudenberg, nahe Utrecht

England: Wodnes dic $>$ Wansdyke ${ }^{3}$

Dänemark: Odense, Onshøj

Norwegen: Odensakr, Othaensweth $>$ Onsved

Schweden: Odensala, Odhisredh $>$ Odensjö $^{4}$

außer auf Island, das während der Jahre 870-930 ,ganz staatenlos “ war ${ }^{5}$ und darum noch alle Odinsnamen entbehrend ${ }^{6}$. Odin wurde von den Landnamsmännern nicht als Himmelsgott, sondern als Gott der Könige und Jarle aufgefaßt (vgl. Hárb. Str. 26).

\section{Der Begriff „Ding der Götter".}

Dieser Begriff spiegelt vor allem den fertigen Götterstaat, Der Ausdruck ping gođa findet sich in Hym. Str. 39, und in Drymskv. Str. I4 wird erzählt, daß alle Götter ,auf dem Ding“ (á pingi) waren. Ungefähr denselben Sinn hat das Wort rokstólar, ,Richterstühle', in Vsp. Str. 6, 9, 23 und 25, und die Angabe, daß die Asen jeden Tag

\footnotetext{
1 Text in Den norsk-isländska skaldediktningen, I, S. 30.

2 G. Dumézil, Leçon inaugural, Paris r950, S. I4.

s Dumézil, L'idéologie tripartie, S. 57 f.

$4 \mathrm{~K}$. Helm, Altgermanische Religionsgeschichte, 2: 2, Heidelberg 1953, S. $175 \mathrm{ff}$.

${ }^{5}$ de Vries, Altgermanische Religionsgeschichte, 2, S. $50 \mathrm{ff}$.

' Genzmer, S. I25.
} 
(Grimn. Str. 30).

Aufhellend in unsrem Zusammenhang ist jetzt, daß diese Götterversammlung von keinem Geringeren als Tjodolf von Hvin als ping pridja bezeichnet wird (Yngl. Str. 32), da pridi gemäß Eilif Godrunarsson ein Name Odins ist (Fórsdr. Str. 2)1. Wir erinnern auch daran, daß das Eidsformular des Westgötagesetzes (oben S. I $_{5}$ ), eben im Zusammenhang mit dem irdischen Ding, das Pantheon durch den Ausdruck holl gudh, ,gnädige Götter" zusammenfaßt.

D. Die Fürstengenealogien.

Karl Hauck hat in einer Reihe von gelehrten Studien 1954-1970 (siehe das Literaturverzeichnis) gezeigt, daß die Könige oft göttliche Namen wie Yngvi bei den Svear, Hendinos unter den Burgundern, Ansis unter den Goten trugen ${ }^{2}$ und daß die germanischen Herrschergenealogien unerwartete und unschätzbare Auskünfte über die Auffassung der origo der Völker und der Normen und Kultmythen bei den Sachsen, Franken u. a. erteilen können. Dadurch ,können die Kulte germanischer Staatlichkeit ... Gegenstand kritischer Quellenforschung sein" ${ }^{\text {"3 }}$, und man erfährt, daß „die genealogische Urgeschichte Mythos, heilige Geschichte ist" ${ }^{\text {" }}$. Das germanische Königsglück ist keine magische oder absolute Erscheinung, sondern „dieses Charisma ist göttliche Begabung" ${ }^{5}$.

Die Genealogien der Northumbrier, Ostanglier, Ynglinger, Skjoldunger u. s. w. gehen zu Odin zurück. Aber ein merkwürdiger Umstand ist nun, daß diese Listen später neue göttliche Genealogien gezeugt haben, die einen organisierten Götterstaat voraussetzen. Das beste Beispiel ist die Ynglingasaga. Der Zweck dieser ist, den Ursprung des neuen norwegischen Gottesstaates aus den Uppsala-Königen mit deren Urahn Odin herzuleiten. In Kap. 9-12 begegnet uns aber eine Erbfolge in dem Götterstaat, die nicht

O. Lárusson, „Kultminne i stadnamn, 4. Island“, Religionshistorie, utg. av Nils Lid (Nordisk kultur, 26), 1942, S. 79.

2 Texte in Den norsk-isländska skaldediktningen, S. 8 und 76.

a Hauck, ,Die geschichtliche Bedeutung", S. Ir2.

"Hauck, ,Lebensnormen", S. 204.

"Hauck, „Lebensnormen“, S. 219. 
nur Njord, Frey und Freyja umfaßt (Kap. 9-10), sondern auch Fjolnir, Sveigdir, seine Frau Vana und alle seine Verwandten in Deutschland und „Groß-Schweden“ zur Götterwelt zählt (Kap. II). Ja, wie ich in meinem Buch „Die Religion der Germanen“ zu zeigen beabsichtige, scheinen auch Vanlandi, Visbur und deren Frauen und Söhne zum mythologischen Bereich zu gehören ${ }^{1}$. Der Gottesstaat auf Erden hat einen Götterstaat im Himmel gezeugt.

So besaßen, während der Zeit, die unsere schriftlichen Quellen umfassen, die Germanen einen doppelten Mythus von dem Staat:

I. den Mythus vom irdischen Gottesstaat mit einer Machtteilung zwischen Herrscher und Ding,

2. den Mythus vom himmlischen Götterstaat mit einer ähnlichen Machtteilung zwischen Odin als pater patrum und den übrigen Göttern.

Das Glied zwischen Gottesstaat und Götterstaat, zwischen Erden und Himmel, ist der sakrale König, der Gipfel des Gottesstaates und der Urenkel des Gipfels des Götterstaates.

\section{Texte}

Cornelius Tacitus Germania. Originalets text med svensk tolkning ... av A. Önnerfors. Stockholm I96r.

Corpus Iuris Sueo-Gotorum Antiqui, ed. C. J. Schlyter, I, Stockholm I827, 6, Lund 1844 .

Edda. Die Lieder des Codex Regius nebst verwandten Denkmälern, hrsg. von G. Neckel Aufl. von H. Kuhn, Heidelberg 1962.

Ílenzk fornrit, 6, Reykjavík I943, 8, I939, 9, 1939, I2, 1954.

Norges gamle love intil 1387 ved R. Keyser-P. A. Munch, $1-2$, Christiania 1846-48.

\section{Verkürzungen}

CISA $=$ Corpus Iuris Sueo-Gotorum Antiqui.

$f F=I_{\text {slen }} k$ forntit.

$N G L=$ Norges gamle love intil 1387.

$R H R=$ Revue de l'histoire des religions.

RegSacr. = La regalità sacra. Contributi al tema dell'VIII congresso internazionale di storia delle religioni (Roma, aprile 1955). Pubblicati col concorso della Giunta Centrale per gli Studi Storici, Roma (Studies in the History of Religions, 4), Leiden I959.

\footnotetext{
${ }^{1}$ Hauck, ,Die geschichtliche Bedeutung“", S. ro3.
} 